\title{
Optimal orientations of strong products of paths
}

\author{
Tjaša Paj Erker \\ University of Maribor, FME, Smetanova 17, 2000 Maribor, Slovenia
}

Received 9 March 2018, accepted 13 July 2018, published online 8 August 2018

\begin{abstract}
Let $\operatorname{diam}_{\min }(G)$ denote the minimum diameter of a strong orientation of $G$ and let $G \otimes H$ denote the strong product of graphs $G$ and $H$. In this paper we prove that $\operatorname{diam}_{\text {min }}\left(P_{m} \otimes P_{n}\right)=\operatorname{diam}\left(P_{m} \otimes P_{n}\right)$ for $m, n \geq 5, m \neq n$, and $\operatorname{diam}_{\min }\left(P_{m} \otimes P_{n}\right)=$ $\operatorname{diam}\left(P_{m} \otimes P_{n}\right)+1$ for $m, n \geq 5, m=n$. We also prove that $\operatorname{diam}_{\min }(G \otimes H) \leq$ $\max \left\{\operatorname{diam}_{\min }(G), \operatorname{diam}_{\min }(H)\right\}$ for any connected bridgeless graphs $G$ and $H$.
\end{abstract}

Keywords: Diameter, strong orientation, strong product.

Math. Subj. Class.: 05C12, 05C76

\section{Introduction}

Let $D=(V(D), A(D))$ be a directed graph. If $(u, v) \in A(D)$, we write $u \rightarrow v$. A $u v$-path is a directed path $u=u_{1} u_{2} \ldots u_{n}=v$ from a vertex $u$ to a vertex $v$. The length of the path $u=u_{1} u_{2} \ldots u_{n}=v$ is $n-1$. If every vertex in $D$ is reachable from every other vertex in $D$, we say that directed graph $D$ is strong (there is a directed $u v$-path in $D$ for every $u, v \in V(D)$ ). The distance from $u$ to $v$ is the length of a shortest directed $u v$-path in $D$, denoted by $\operatorname{dist}_{D}(u, v)$. The greatest distance among all pairs of vertices in $D$ is the diameter of $D$, so

$$
\operatorname{diam}(D)=\max \left\{\operatorname{dist}_{D}(u, v) \mid u, v \in V(D)\right\} .
$$

Note that the distance of two vertices $u, v$ in undirected graph $G, \operatorname{dist}_{G}(u, v)$, is the length of a shortest undirected $u v$-path in $G$ and the greatest distance between any two vertices in $G$ is the diameter of $G$, denoted by $\operatorname{diam}(G)$.

Let $G$ be an undirected graph. An orientation of $G$ is a digraph $D$ obtained from $G$ by assigning to each edge in $G$ a direction. Let $\mathcal{D}(G)$ denote the family of all strong orientations of $G$. In [9] it is proved that every connected bridgeless graph admits a strong orientation. We define the minimum diameter of a strong orientation of $G$ as

$$
\operatorname{diam}_{\min }(G)=\min \{\operatorname{diam}(D) \mid D \in \mathcal{D}(G)\} .
$$

E-mail address: tjasa.paj@um.si (Tjaša Paj Erker) 
The parameter $\operatorname{diam}_{\min }(G)$ was studied by many authors, because it is important from theoretical and practical points of view, as an application in traffic control problems. Orientations of graphs can be viewed as arrangements of one-way streets, if $G$ is thought of as the system of two-way streets in a city, and we want to make every street in the city one-way and still get from every point to every other point (see $[9,10])$.

For every bridgeless connected graph $G$ of radius $r$ it was shown, see [1], that $\operatorname{diam}_{\min }(G) \leq 2 r^{2}+2 r$. There were also some determined values of the minimum diameter of a strong orientation of the Cartesian product of graphs. For Cartesian product of two paths it was proved that $\operatorname{diam}_{\min }\left(P_{m} \square P_{n}\right)=\operatorname{diam}\left(P_{m} \square P_{n}\right)$, for $m \geq 3$ and $n \geq 6$, see [5]. In [8] it was proved that $\operatorname{diam}_{\min }\left(C_{m} \square C_{n}\right)=\operatorname{diam}\left(C_{m} \square C_{n}\right)$ for $m, n \geq 6$. In [7] Koh and Tay proved that $\operatorname{diam}_{\min }\left(T_{1} \square T_{2}\right)=\operatorname{diam}\left(T_{1} \square T_{2}\right)$ for trees $T_{1}$ and $T_{2}$ with diameters at least 4 . They also studied the diameter of orientations of $K_{m} \square K_{n}, K_{m} \square P_{n}$, $P_{m} \square C_{n}$ and $K_{m} \square C_{n}$ (see [4, 5, 6]).

In [3], the upper bound for the strong radius and the strong diameter of Cartesian product of graphs are determined.

In this article we consider the minimum diameter of strong orientations of strong products of graphs. The strong product of graphs $G$ and $H$ is the graph, denoted by $G \otimes H$, with the vertex set $V(G \otimes H)=V(G) \times V(H)$ where two distinct vertices $(u, v)$ and $\left(u^{\prime}, v^{\prime}\right)$ are adjacent in $G \otimes H$ if and only if $u u^{\prime} \in E(G)$ and $v=v^{\prime}$, or $u=u^{\prime}$ and $v v^{\prime} \in E(H)$, or $u u^{\prime} \in E(G)$ and $v v^{\prime} \in E(H)$. For $v \in V(H)$ we define the $G$-layer $G_{v}$ :

$$
G_{v}=\{(u, v) \mid u \in V(G)\} .
$$

Analogously we define $H$-layers.

In the next section we prove that $\operatorname{diam}_{\min }\left(P_{m} \otimes P_{n}\right)=\operatorname{diam}\left(P_{m} \otimes P_{n}\right)$, for $m, n \geq 5$, $m \neq n$ and that $\operatorname{diam}_{\text {min }}\left(P_{m} \otimes P_{n}\right)=\operatorname{diam}\left(P_{m} \otimes P_{n}\right)+1$, for $m, n \geq 5, m=n$.

\section{Orientations of $\boldsymbol{P}_{m} \otimes \boldsymbol{P}_{n}$}

In [7] Koh and Tay proved that $\operatorname{diam}_{\min }\left(P_{m} \square P_{n}\right)=\operatorname{diam}\left(P_{m} \square P_{n}\right)$, for $m \geq 5$ and $n \geq 5$. We use some of their notations. So we will define four sections of $V\left(P_{m} \otimes P_{n}\right)$ and two basic orientations of $P_{s} \otimes P_{t}$, where $s, t \geq 3$, similarly as it was introduced in [7]. For $m, n \geq 5$ we define

(i) Southwest Section $\mathrm{SW}=\left\{(i, j) \mid 1 \leq i \leq\left\lceil\frac{m}{2}\right\rceil, 1 \leq j \leq\left\lceil\frac{n}{2}\right\rceil\right\}$;

(ii) Northwest Section NW $=\left\{(i, j) \mid 1 \leq i \leq\left\lceil\frac{m}{2}\right\rceil,\left\lceil\frac{n+1}{2}\right\rceil \leq j \leq n\right\}$;

(iii) Southeast Section $\mathrm{SE}=\left\{(i, j) \mid\left\lceil\frac{m+1}{2}\right\rceil \leq i \leq m, 1 \leq j \leq\left\lceil\frac{n}{2}\right\rceil\right\}$;

(iv) Northeast Section $\mathrm{NE}=\left\{(i, j) \mid\left\lceil\frac{m+1}{2}\right\rceil \leq i \leq m,\left\lceil\frac{n+1}{2}\right\rceil \leq j \leq n\right\}$.

We define two basic orientations of $P_{s} \otimes P_{t}$, where $s, t \geq 3$ : if $s \leq t$, we define the orientation $F_{1}$ of $P_{s} \otimes P_{t}$ as:

(i) For $1 \leq i \leq s-1$ and $2 \leq j \leq t,(i, j) \rightarrow(i+1, j-1)$;

(ii) For $1 \leq i \leq s-1$ and $1 \leq j \leq t-1,(i+1, j+1) \rightarrow(i, j)$ if $j-i \geq t-s$ and $(i, j) \rightarrow(i+1, j+1)$ if $j-i<t-s$

(iii) For $1 \leq i \leq s-1$ and $2 \leq j \leq t,(i, j) \rightarrow(i, j-1)$;

(iv) For $1 \leq j \leq t-1,(s, j) \rightarrow(s, j+1)$; 
(v) For $1 \leq i \leq s-1$ and $1 \leq j \leq t-1,(i, j) \rightarrow(i+1, j)$;

(vi) For $2 \leq i \leq s,(i, t) \rightarrow(i-1, t)$;

and if $s>t$, we define the orientation $F_{2}$ of $P_{s} \otimes P_{t}$ as:

(i) For $2 \leq i \leq s$ and $1 \leq j \leq t-1,(i, j) \rightarrow(i-1, j+1)$;

(ii) For $1 \leq i \leq s$ and $1 \leq j \leq t,(i+1, j+1) \rightarrow(i, j)$ if $i-j \geq s-t$ and $(i, j) \rightarrow(i+1, j+1)$ if $i-j<s-t$

(iii) For $1 \leq i \leq s-1$ and $1 \leq j \leq t-1,(i, j) \rightarrow(i, j+1)$;

(iv) For $2 \leq j \leq t,(s, j) \rightarrow(s, j-1)$;

(v) For $2 \leq i \leq s$ and $1 \leq j \leq t-1,(i, j) \rightarrow(i-1, j)$;

(vi) For $1 \leq i \leq s-1,(i, t) \rightarrow(i+1, t)$.

The orientation $F_{1}$ of $P_{3} \otimes P_{4}$ and the orientation $F_{2}$ of $P_{4} \otimes P_{3}$ is shown in Figure 1.
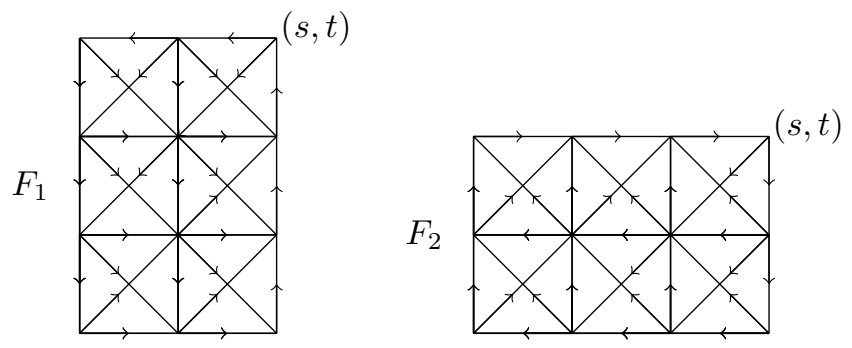

Figure 1: Orientations $F_{1}$ and $F_{2}$.

Observation 2.1. If $s<t$, for any $(i, j) \in V\left(F_{1}\right)$, $\operatorname{dist}_{F_{1}}((i, j),(s, t-1)) \leq t-2$.

Proof. Let $(i, j) \in V\left(F_{1}\right)$. We shall consider four cases.

(i) If $j \neq t$ and $j \geq i+t-s-1$, then $(i, j) \rightarrow(i+1, j) \rightarrow \cdots \rightarrow(j-(t-s)+1, j) \rightarrow$ $(j-(t-s)+2, j+1) \rightarrow \cdots \rightarrow(s, t-1)$ is a path of length at most $s-1 \leq t-2$.

(ii) If $j \neq t$ and $j<i+t-s-1$, then $(i, j) \rightarrow(i+1, j+1) \rightarrow \cdots \rightarrow(s, j+s-i) \rightarrow$ $(s, j+s-i+1) \rightarrow \cdots \rightarrow(s, t-1)$ is a path of length at most $t-2$.

(iii) If $j=t$ and $i \neq s$, then $(i, t) \rightarrow(i+1, t-1) \rightarrow(i+2, t-1) \rightarrow \cdots \rightarrow(s, t-1)$ is a path of length at most $s-1 \leq t-2$.

(iv) If $j=t$ and $i=s$, then $(s, t) \rightarrow(s-1, t-1) \rightarrow(s, t-1)$ is a path of length two.

Observation 2.2. If $s<t$, for any $(i, j) \in V\left(F_{1}\right)$, $\operatorname{dist}_{F_{1}}((i, j),(s, t)) \leq t-1$.

Proof. Since $(s, t-1) \rightarrow(s, t)$, the claim follows by Observation 2.1:

$$
\operatorname{dist}_{F_{1}}((i, j),(s, t))=\operatorname{dist}_{F_{1}}((i, j),(s, t-1))+1 \leq s-1+1 \leq t-1 .
$$

Observation 2.3. If $s<t$, for any $(i, j) \in V\left(F_{1}\right)$, $\operatorname{dist}_{F_{1}}((s-1, t),(i, j)) \leq t-1$. 
Proof. Let $(i, j) \in V\left(F_{1}\right)$. We shall consider four cases.

(i) If $i \neq s$ and $j>i+t-s$, then $(s-1, t) \rightarrow(s-2, t) \rightarrow \cdots \rightarrow(i+(t-j), t) \rightarrow$ $(i+(t-j)-1, t-1) \rightarrow \cdots \rightarrow(i, j)$ is a path of length at most $s-2 \leq t-2$.

(ii) If $i \neq s$ and $j \leq i+t-s$, then $(s-1, t) \rightarrow(s-1, t-1) \rightarrow(s-2, t-2) \rightarrow \cdots \rightarrow$ $(i, i+t-s) \rightarrow(i, i+t-s-1) \rightarrow \cdots \rightarrow(i, j)$ is a path of length at most $t-1$.

(iii) If $i=s$ and $j \neq t,(s-1, t) \rightarrow(s-1, t-1) \rightarrow(s-1, t-2) \rightarrow \cdots \rightarrow$ $(s-1, j+1) \rightarrow(s, j)$ is a path of length at most $t-1$.

(iv) If $i=s$ and $j=t$, then $(s-1, t) \rightarrow(s, t-1) \rightarrow(s, t)$ is a path of length two.

Observation 2.4. If $s<t$, for any $(i, j) \in V\left(F_{1}\right)$ dist $_{F_{1}}((s, t),(i, j)) \leq t-1$.

Proof. Since $(s, t) \rightarrow(s-1, t)$ and $(s, t) \rightarrow(s-1, t-1)$, the proof is similar as the proof of Observation 2.3.

Observation 2.5. If $s=t$, for any $(i, j) \in V\left(F_{1}\right)$ dist $_{F_{1}}((i, j),(s, s)) \leq s$.

Proof. Let $(i, j) \in V\left(F_{1}\right)$. We shall consider three cases.

(i) If $j \neq t$ and $j \geq i-1$, then $(i, j) \rightarrow(i+1, j) \rightarrow \cdots \rightarrow(j+1, j) \rightarrow(j+2, j+1) \rightarrow$ $\cdots \rightarrow(s, s-1) \rightarrow(s, s)$ is a path of length at most $s$.

(ii) If $j \neq t$ and $j<i-1$, then $(i, j) \rightarrow(i+1, j+1) \rightarrow \cdots \rightarrow(s, j+s-i) \rightarrow$ $(s, j+s-i+1) \rightarrow \cdots \rightarrow(s, s)$ is a path of length at most $s-1$.

(iii) If $j=s$ and $i \neq s$, then $(i, s) \rightarrow(i+1, s-1) \rightarrow(i+2, s-1) \rightarrow \cdots \rightarrow(s, s-1) \rightarrow$ $(s, s)$ is a path of length at most $s$.

Observation 2.6. If $s=t$, for any $(i, j) \in V\left(F_{1}\right)$, $\operatorname{dist}_{F_{1}}((s, s),(i, j)) \leq s-1$.

Proof. Let $(i, j) \in V\left(F_{1}\right)$. We shall consider three cases.

(i) If $i \neq s$ and $j>i$, then $(s, s) \rightarrow(s-1, s) \rightarrow \cdots \rightarrow(i+(s-j), s) \rightarrow$ $(i+(s-j)-1, t-1) \rightarrow \ldots \rightarrow(i, j)$ is a path of length at most $s-1$.

(ii) If $i \neq s$ and $j \leq i$, then $(s, s) \rightarrow(s-1, s-1) \rightarrow \cdots \rightarrow(i, i) \rightarrow(i, i-1) \rightarrow$ $\ldots \rightarrow(i, j)$ is a path of length at most $s-1$.

(iii) If $i=s$ and $j \neq s-1,(s, s) \rightarrow(s-1, s-1) \rightarrow(s-1, s-2) \rightarrow \cdots \rightarrow$ $(s-1, j+1) \rightarrow(s, j)$ is a path of length at most $s-1$.

(iv) If $i=s$ and $j=s-1$, then $(s, s) \rightarrow(s-1, s-1) \rightarrow(s, s-1)$ is a path of length two.

Similarly as above, we can prove next Observations 2.7-2.10.

Observation 2.7. If $s>t$, for any $(i, j) \in V\left(F_{2}\right), \operatorname{dist}_{F_{2}}((s, t-1),(i, j)) \leq s-1$.

Observation 2.8. If $s>t$, for any $(i, j) \in V\left(F_{2}\right)$ dist $_{F_{2}}((s, t),(i, j)) \leq s-1$.

Observation 2.9. If $s>t$, for any $(i, j) \in V\left(F_{2}\right), \operatorname{dist}_{F_{2}}((i, j),(s-1, t)) \leq s-2$.

Observation 2.10. If $s>t$, for any $(i, j) \in V\left(F_{2}\right) \operatorname{dist}_{F_{2}}((i, j),(s, t)) \leq s-1$. 
In [7], Koh and Tay also introduced a key-vertex $v \in V(F)$ of digraph $F$. Let $F \in$ $\mathcal{D}\left(P_{s} \otimes P_{t}\right)$. We say that a vertex $v \in V(F)$ is a key-vertex of $F$ if

$$
\operatorname{dist}_{F}(u, v) \leq \max \{t, s\} \quad \text { and } \quad \operatorname{dist}_{F}(v, u) \leq \max \{t, s\}
$$

for all $u \in V(F)$. Note that $(s, t)$ is a key-vertex of $F_{1}$ and of $F_{2}$.

Analogously as $F_{1}$ and $F_{2}$, we define 6 other isomorphic orientations $F_{i}, 3 \leq i \leq 8$ of $P_{s} \otimes P_{t}$ as shown in Figures 2 and 3.
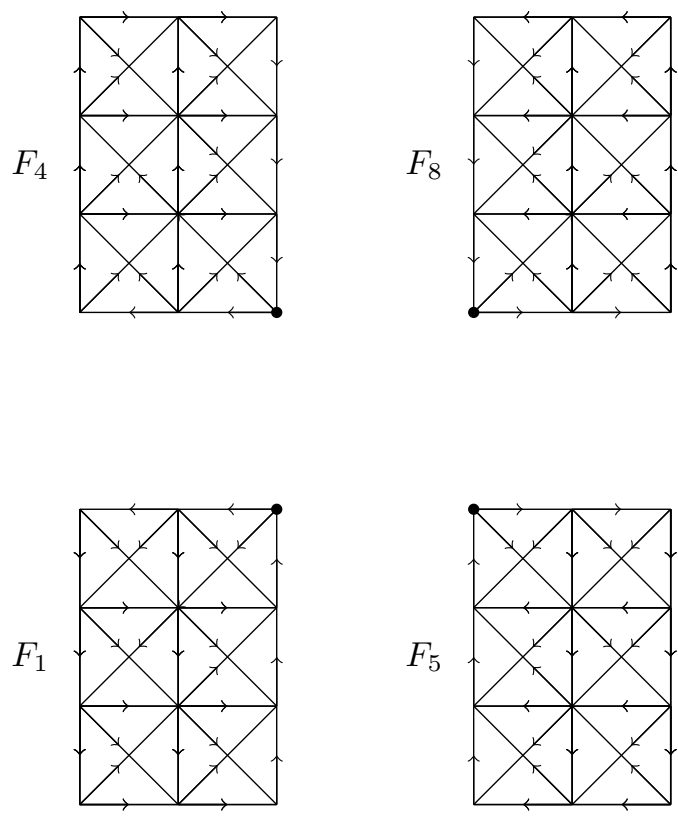

Figure 2: Orientations $F_{1}, F_{4}, F_{5}$ and $F_{8}$.

Obviously vertices denoted by black dots in Figures 2 and 3 are key-vertices of $F_{i}$ for $i=1, \ldots, 8$ (similar arguments as in Observations 2.1-2.6).

Lemma 2.11. Let $m, n \geq 5, m \neq n$ and $m, n \equiv 1(\bmod 2)$. Then

$$
\operatorname{diam}_{\min }\left(P_{m} \otimes P_{n}\right) \leq \max \{m-1, n-1\} .
$$

Proof. Let $m<n$. We define the orientation $D$ of $P_{m} \otimes P_{n}$ by $F_{1}, F_{4}, F_{5}$ and $F_{8}$ :

(a) orient the section $\mathrm{NW}$ as $F_{4}$;

(b) orient the section $\mathrm{NE}$ as $F_{8}$;

(c) orient the section $\mathrm{SW}$ as $F_{1}$;

(d) orient the section $\mathrm{SE}$ as $F_{5}$.

As an illustration, the orientation of $P_{5} \otimes P_{7}$ is shown in Figure 4. The vertex $z=$ $\left(\frac{m+1}{2}, \frac{n+1}{2}\right)$ is the key-vertex of each $F_{i}$, for $i=1,4,5,8$. For any $u, v \in V(D)$,

$$
\operatorname{dist}_{D}(u, v) \leq \operatorname{dist}_{D}(u, z)+\operatorname{dist}_{D}(z, v) .
$$



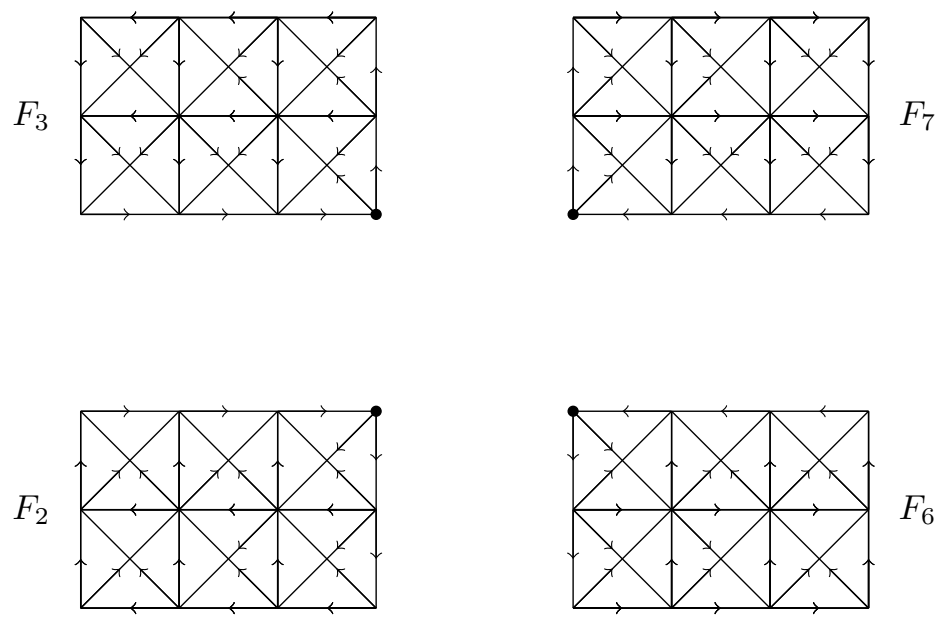

Figure 3: Orientations $F_{2}, F_{3}, F_{6}$ and $F_{7}$.

Since $\operatorname{dist}_{D}(u, z) \leq \frac{n-1}{2}$ and $\operatorname{dist}_{D}(z, v) \leq \frac{n-1}{2}$ (similarly as in Observation 2.2 and Observation 2.4), we have

$$
\operatorname{dist}_{D}(u, v) \leq \frac{n-1}{2}+\frac{n-1}{2}=n-1 .
$$

If $m>n$ we define the orientation $D$ of $P_{m} \otimes P_{n}$ by $F_{2}, F_{3}, F_{6}$ and $F_{7}$. Similarly as above, we have

$$
\operatorname{dist}_{D}(u, v) \leq \operatorname{dist}_{D}(u, z)+\operatorname{dist}_{D}(z, v) \leq \frac{m-1}{2}+\frac{m-1}{2}=m-1
$$

(see Observation 2.10 and Observation 2.8).

Lemma 2.12. Let $m, n \geq 6, m \neq n$ and $m, n \equiv 0(\bmod 2)$. Then

$$
\operatorname{diam}_{\min }\left(P_{m} \otimes P_{n}\right) \leq \max \{m-1, n-1\}
$$

Proof. Let $m<n$. Denote $z_{1}=\left(\frac{m}{2}, \frac{n}{2}\right), z_{4}=\left(\frac{m}{2}, \frac{n}{2}+1\right), z_{5}=\left(\frac{m}{2}+1, \frac{n}{2}\right)$ and $z_{8}=\left(\frac{m}{2}+1, \frac{n}{2}+1\right)$. We define the orientation $D$ of $P_{m} \otimes P_{n}$ by $F_{1}, F_{4}, F_{5}$ and $F_{8}$ as follows:

(a) orient the section $\mathrm{NW}$ as $F_{4}$;

(b) orient the section $\mathrm{NE}$ as $F_{8}$;

(c) orient the section $\mathrm{SW}$ as $F_{1}$;

(d) orient the section $\mathrm{SE}$ as $F_{5}$;

(e) Orient $z_{1} \rightarrow\left(\frac{m}{2}-1, \frac{n}{2}+1\right),\left(\frac{m}{2}+1, \frac{n}{2}-1\right) \rightarrow z_{1}, z_{4} \rightarrow\left(\frac{m}{2}-1, \frac{n}{2}\right),\left(\frac{m}{2}+1, \frac{n}{2}+2\right) \rightarrow$ $z_{4}, z_{5} \rightarrow\left(\frac{m}{2}+2, \frac{n}{2}+1\right),\left(\frac{m}{2}, \frac{n}{2}-1\right) \rightarrow z_{5}, z_{8} \rightarrow\left(\frac{m}{2}+2, \frac{n}{2}\right),\left(\frac{m}{2}, \frac{n}{2}+2\right) \rightarrow z_{8}$, and orient all other edges arbitrarily. 


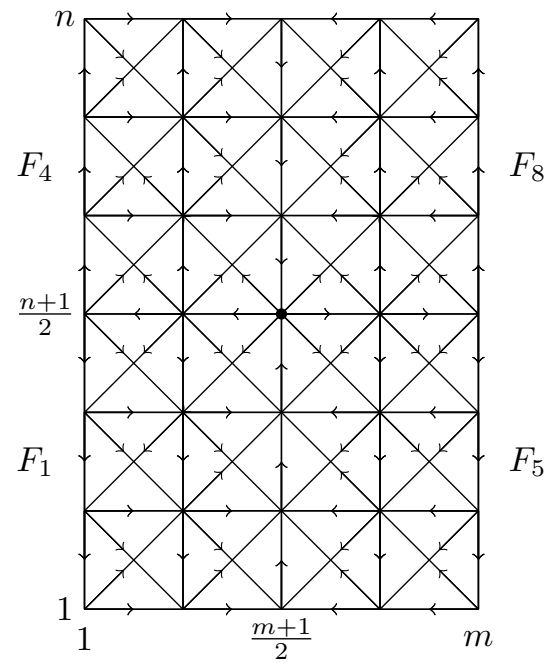

Figure 4: The orientation $D$ of $P_{5} \otimes P_{7}$.

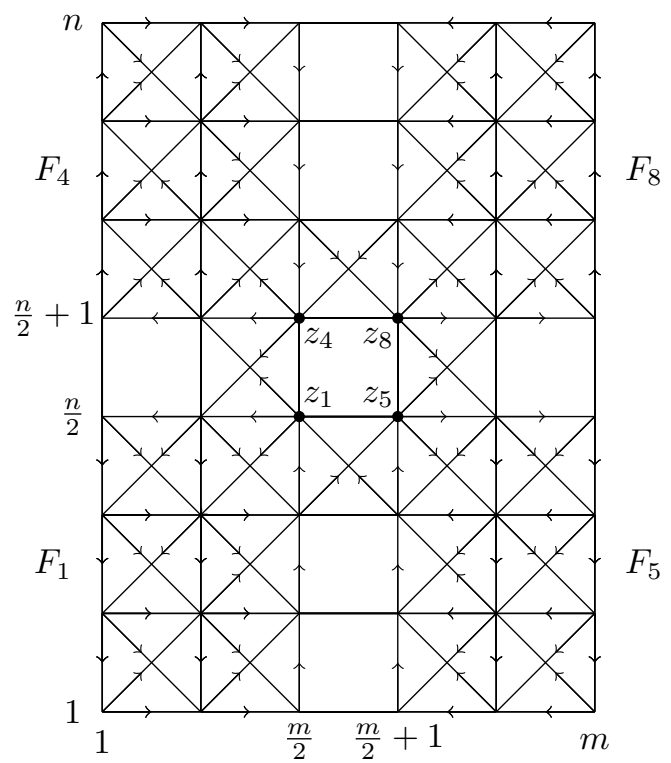

Figure 5: The orientation $D$ of $P_{6} \otimes P_{8}$. 
The orientation $D$ is shown in Figure 5. Note that vertices $z_{1}, z_{4}, z_{5}$ and $z_{8}$ are key-vertices of $F_{i}$, for $i=1,4,5,8$.

Let $u, v \in V(D)$. We claim that $\operatorname{dist}_{D}(u, v) \leq n-1$. There are four cases.

(i) If $u$ and $v$ are in the same section, then we have

$$
\operatorname{dist}_{D}(u, v) \leq \operatorname{dist}_{D}\left(u, z_{i}\right)+\operatorname{dist}_{D}\left(z_{i}, v\right) \leq \frac{n}{2}-1+\frac{n}{2}-1=n-2
$$

as in Observation 2.2 and Observation 2.4.

(ii) If $u \in \mathrm{NW}$ and $v \in \mathrm{SW}$, then (see Observation 2.2 and Observation 2.3):

$$
\begin{aligned}
\operatorname{dist}_{D}(u, v) & \leq \operatorname{dist}_{D}\left(u, z_{4}\right)+\operatorname{dist}_{D}\left(z_{4},\left(\frac{m}{2}-1, \frac{n}{2}\right)\right)+\operatorname{dist}_{D}\left(\left(\frac{m}{2}-1, \frac{n}{2}\right), v\right) \\
& \leq \frac{n}{2}-1+1+\frac{n}{2}-1=n-1 .
\end{aligned}
$$

The argument is similar if $u \in \mathrm{SW}$ and $v \in \mathrm{NW}$, or $u \in \mathrm{NE}$ and $v \in \mathrm{SE}$, or $u \in \mathrm{SE}$ and $v \in \mathrm{NE}$.

(iii) If $u \in \mathrm{SW}$ and $v \in \mathrm{SE}$, then the claim follows from Observation 2.1 and Observation 2.4, similarly as above. Also, if $u \in \mathrm{SE}$ and $v \in \mathrm{SW}$, or $u \in \mathrm{NW}$ and $v \in \mathrm{NE}$, or $u \in \mathrm{NE}$ and $v \in \mathrm{NW}$, then the argument is analogous.

(iv) If $u \in \mathrm{SW}$ and $v \in \mathrm{NE}$, then (see Observation 2.1 and Observation 2.3) we have

$$
\begin{aligned}
\operatorname{dist}_{D}(u, v) \leq & \operatorname{dist}_{D}\left(u,\left(\frac{m}{2}, \frac{n}{2}-1\right)\right)+\operatorname{dist}_{D}\left(\left(\frac{m}{2}, \frac{n}{2}-1\right), z_{5}\right)+ \\
& \quad+\operatorname{dist}_{D}\left(z_{5},\left(\frac{m}{2}+2, \frac{n}{2}+1\right)\right)+\operatorname{dist}_{D}\left(\left(\frac{m}{2}+2, \frac{n}{2}+1\right), v\right) \\
\leq & \frac{n}{2}-2+1+1+\frac{n}{2}-1=n-1 .
\end{aligned}
$$

The argument is similar for $u \in \mathrm{NE}$ and $v \in \mathrm{SW}$, or $u \in \mathrm{NW}$ and $v \in \mathrm{SE}$, or $u \in \mathrm{SE}$ and $v \in \mathrm{NW}$.

Analogously if $m>n$, we have $\operatorname{dist}_{D}(u, v) \leq m-1$ for any $u, v \in V(D)$.

Lemma 2.13. Let $m \geq 5, n \geq 6, m \equiv 1(\bmod 2)$ and $n \equiv 0(\bmod 2)$. Then

$$
\operatorname{diam}_{\min }\left(P_{m} \otimes P_{n}\right) \leq \max \{m-1, n-1\} .
$$

Proof. Let $m<n$. Denote $z_{1}=\left(\frac{m+1}{2}, \frac{n}{2}\right)$ and $z_{4}=\left(\frac{m+1}{2}, \frac{n}{2}+1\right)$. We define the orientation $D$ of $P_{m} \otimes P_{n}$ by $F_{1}, F_{4}, F_{5}$ and $F_{8}$ as follows:

(a) orient the section $\mathrm{NW}$ as $F_{4}$;

(b) orient the section $\mathrm{NE}$ as $F_{8}$;

(c) orient the section $\mathrm{SW}$ as $F_{1}$;

(d) orient the section $\mathrm{SE}$ as $F_{5}$;

(e) orient $z_{4} \rightarrow\left(\frac{m+1}{2}-1, \frac{n}{2}\right), z_{1} \rightarrow z_{4}, z_{4} \rightarrow\left(\frac{m+1}{2}+1, \frac{n}{2}\right)$, and orient all other edges arbitrarily.

The orientation $D$ is shown in Figure 6. Note that vertex $z_{1}$ is a key-vertex of $F_{1}$ and $F_{5}$ and that vertex $z_{4}$ is a key-vertex of $F_{4}$ and $F_{8}$. 


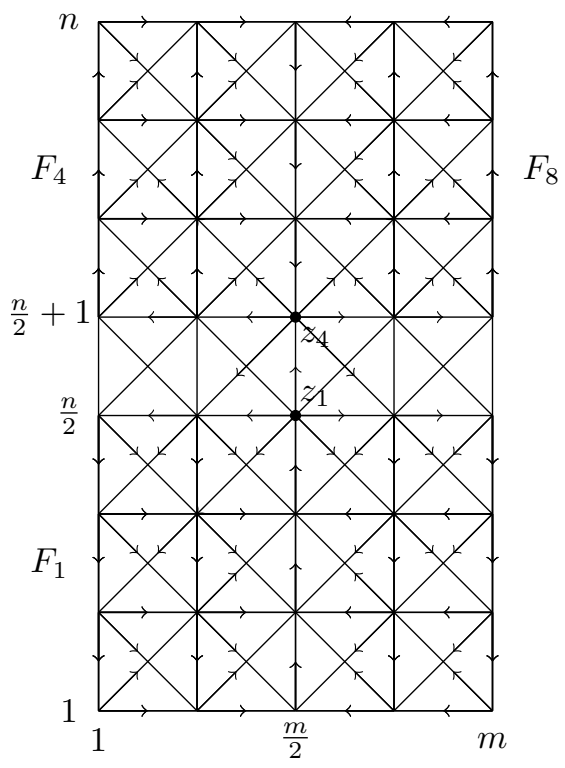

Figure 6: The orientation $D$ of $P_{5} \otimes P_{8}$.

Let $u, v \in V(D)$. There are three cases.

(i) If $u \in \mathrm{NW} \cup \mathrm{NE}$ and $v \in \mathrm{NW} \cup \mathrm{NE}$, then we have

$$
\operatorname{dist}_{D}(u, v) \leq \operatorname{dist}_{D}\left(u, z_{4}\right)+\operatorname{dist}_{D}\left(z_{4}, v\right) \leq \frac{n}{2}-1+\frac{n}{2}-1=n-2
$$

(see Observation 2.2 and Observation 2.4). The case that $\{u, v\} \subseteq \mathrm{SW} \cup \mathrm{SE}$ is similar.

(ii) If $u \in \mathrm{SW} \cup \mathrm{SE}$ and $v \in \mathrm{NW} \cup \mathrm{NE}$, then (see Observation 2.2 and Observation 2.4):

$$
\begin{aligned}
\operatorname{dist}_{D}(u, v) & \leq \operatorname{dist}_{D}\left(u, z_{1}\right)+\operatorname{dist}_{D}\left(z_{1}, z_{4}\right)+\operatorname{dist}_{D}\left(z_{4}, v\right) \\
& \leq \frac{n}{2}-1+1+\frac{n}{2}-1=n-1
\end{aligned}
$$

(iii) If $u \in \mathrm{NW} \cup \mathrm{NE}$ and $v \in \mathrm{SW}$, then from Observation 2.2 and Observation 2.3:

$$
\begin{aligned}
\operatorname{dist}_{D}(u, v) \leq & \operatorname{dist}_{D}\left(u, z_{4}\right)+\operatorname{dist}_{D}\left(z_{4},\left(\frac{m+1}{2}-1, \frac{n}{2}\right)\right)+ \\
& +\operatorname{dist}_{D}\left(\left(\frac{m+1}{2}-1, \frac{n}{2}\right), v\right) \\
\leq & \frac{n}{2}-1+1+\frac{n}{2}-1=n-1 .
\end{aligned}
$$

The case that $u \in \mathrm{NW} \cup \mathrm{NE}$ and $v \in \mathrm{SE}$ is similar.

Let $m>n$. Denote $z_{2}=\left(\frac{m+1}{2}, \frac{n}{2}\right)$ and $z_{3}=\left(\frac{m+1}{2}, \frac{n}{2}+1\right)$. We define the orientation $D$ of $P_{m} \otimes P_{n}$ by $F_{2}, F_{3}, F_{6}$ and $F_{7}$ as follows:

(a) orient the section $\mathrm{NW}$ as $F_{3}$; 
(b) orient the section $\mathrm{NE}$ as $F_{7}$;

(c) orient the section $\mathrm{SW}$ as $F_{2}$;

(d) orient the section $\mathrm{SE}$ as $F_{6}$;

(e) orient $\left(\frac{m+1}{2}-1, \frac{n}{2}\right) \rightarrow z_{3}, z_{3} \rightarrow z_{2},\left(\frac{m+1}{2}+1, \frac{n}{2}\right) \rightarrow z_{3}$ and all other edges oriented arbitrarily.

The rest of the proof is analogously as above.

Note that if $m \geq 5$ and $n \geq 6, m \equiv 0(\bmod 2)$ and $n \equiv 1(\bmod 2)$, we also have (2.1).

Lemma 2.14. Let $m \geq 5, m \equiv 1(\bmod 2)$. Then

$$
\operatorname{diam}_{\min }\left(P_{m} \otimes P_{m}\right) \leq m .
$$

Proof. Denote $z=\left(\frac{m+1}{2}, \frac{m+1}{2}\right)$. We define the orientation $D$ of $P_{m} \otimes P_{m}$ by $F_{1}, F_{4}, F_{5}$ and $F_{8}$ as follows:

(a) orient the section $\mathrm{NW}$ as $F_{4}$;

(b) orient the section $\mathrm{NE}$ as $F_{8}$;

(c) orient the section $\mathrm{SW}$ as $F_{1}$;

(d) orient the section $\mathrm{SE}$ as $F_{5}$.

Note that $z$ is a key-vertex of $F_{i}$, for $i=1,4,5,8$. For any $u, v \in D$ we have

$$
\operatorname{dist}_{D}(u, v) \leq \operatorname{dist}_{D}(u, z)+\operatorname{dist}_{D}(z, v) \leq \frac{m+1}{2}+\frac{m-1}{2}=m
$$

as in Observation 2.5 and Observation 2.6.

Lemma 2.15. Let $m \geq 6, m \equiv 0(\bmod 2)$. Then

$$
\operatorname{diam}_{\text {min }}\left(P_{m} \otimes P_{m}\right) \leq m .
$$

Proof. The proof is similarly as the proof of Lemma 2.12 (it follows from Observations 2.1, 2.3, 2.5 and 2.6).

In [2], it is proved that if $(u, v)$ and $\left(u^{\prime}, v^{\prime}\right)$ are vertices of a strong product $G \otimes H$, then

$$
\operatorname{dist}_{G \bowtie H}\left((u, v),\left(u^{\prime}, v^{\prime}\right)\right)=\max \left\{\operatorname{dist}_{G}\left(u, u^{\prime}\right), \operatorname{dist}_{H}\left(v, v^{\prime}\right)\right\} .
$$

Since $\operatorname{diam}\left(P_{m}\right)=m-1$, we get $\operatorname{diam}\left(P_{m} \otimes P_{n}\right)=\max \{m-1, n-1\}$. Since $\operatorname{diam}\left(P_{m} \otimes P_{n}\right)=\operatorname{dist}_{P_{m} \otimes P_{m}}((1,1),(m, m))=m-1$ and there is only one path from $(1,1)$ to $(m, m)$ in $P_{m} \otimes P_{m}$ possessing the length $m-1$, it follows that

$$
\operatorname{dist}_{D}((1,1),(m, m))>m-1 \quad \text { or } \quad \operatorname{dist}_{D}((m, m),(1,1))>m-1
$$

for any $D \in \mathcal{D}\left(P_{m} \otimes P_{n}\right)$. To combine these two observations with Lemmas 2.11-2.15, we obtain the following theorem: 
Theorem 2.16. If $m, n \geq 5$, then

$$
\operatorname{diam}_{\text {min }}\left(P_{m} \otimes P_{n}\right)= \begin{cases}\operatorname{diam}\left(P_{m} \otimes P_{n}\right), & \text { if } m \neq n ; \\ \operatorname{diam}\left(P_{m} \otimes P_{n}\right)+1, & \text { if } m=n .\end{cases}
$$

At the end of this section, we give the bounds of $\operatorname{diam}_{\min }\left(P_{n} \otimes P_{m}\right)$ for $m<5$. From Figure 7, we see that $n-1 \leq \operatorname{diam}_{\min }\left(P_{n} \otimes P_{2}\right)=n$ for $n>2, n-1 \leq$ $\operatorname{diam}_{\text {min }}\left(P_{n} \otimes P_{3}\right)=n$ for $n>3$ and $n-1 \leq \operatorname{diam}_{\text {min }}\left(P_{n} \otimes P_{4}\right)=n+1$ for $n>4$.
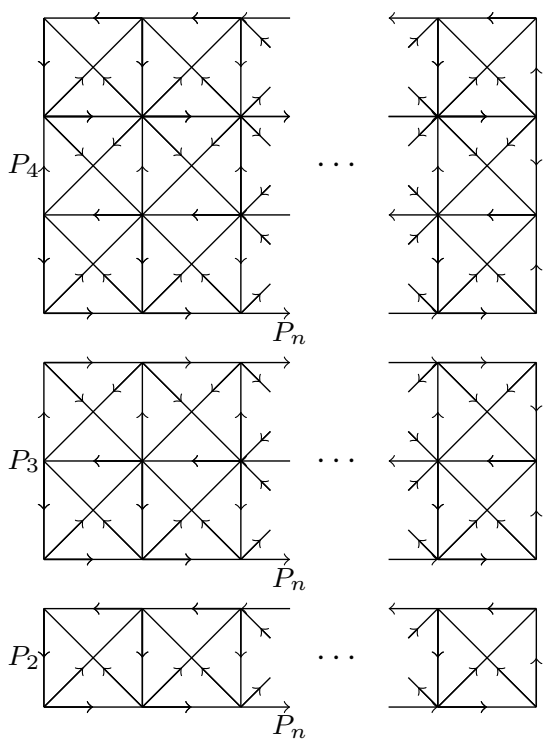

Figure 7: Orientations of $P_{n} \otimes P_{2}, P_{n} \otimes P_{3}$ and $P_{n} \otimes P_{4}$.

\section{Strong orientation of graphs}

In this section we shall prove the next theorem.

Theorem 3.1. Let $G$ and $H$ be connected bridgeless graphs. Then

$$
\operatorname{diam}_{\min }(G \otimes H) \leq \max \left\{\operatorname{diam}_{\min }(G), \operatorname{diam}_{\min }(H)\right\} .
$$

Proof. Let $D_{G}$ be a strong orientation of $G$ such that $\operatorname{diam}\left(D_{G}\right)=\operatorname{diam}_{\min }(G)=d_{1}$ and let $D_{H}$ be a strong orientation of $H$ such that $\operatorname{diam}\left(D_{H}\right)=\operatorname{diam}_{\min }(H)=d_{2}$. We define the orientation $D_{G \otimes H}$ of $G \otimes H$ as:

(a) Every edge with endvertices in layers $G_{v}, v \in V(H)$ gets the orientation $D_{G}$.

(b) Every edge with endvertices in layers $H_{u}, u \in V(G)$ gets the orientation $D_{H}$.

(c) If $u \rightarrow u^{\prime}$ in $G$ and $v \rightarrow v^{\prime}$ in $H$, then $(u, v) \rightarrow\left(u^{\prime}, v^{\prime}\right)$, all other edges are oriented arbitrarily. 
We have to prove that for every pair of vertices $(u, v),\left(u^{\prime}, v^{\prime}\right)$ in $G \otimes H$ there is a directed path $P$ from $(u, v)$ to $\left(u^{\prime}, v^{\prime}\right)$ in $D_{G \otimes H}$, such that the length of $P$ is at most $\max \left\{d_{1}, d_{2}\right\}$.

If $(u, v)$ and $\left(u^{\prime}, v\right)$ are vertices in the same $G$-layer or if $(u, v)$ and $\left(u, v^{\prime}\right)$ are vertices in the same $H$-layer, then there is a directed path from $(u, v)$ to $\left(u^{\prime}, v\right)$ in $D_{G \otimes H}$ of length at most $d_{1}$ or a directed path from $(u, v)$ to $\left(u, v^{\prime}\right)$ of length at most $d_{2}$.

Now let $(u, v)$ and $\left(u^{\prime}, v^{\prime}\right)$ be arbitrary vertices in $D_{G \otimes H}$. There is a directed path $u=$ $u_{1} u_{2} \ldots u_{m}=u^{\prime}$ in $G$ of length at most $d_{1}$ and there is a directed path $v=v_{1} v_{2} \ldots v_{n}=v^{\prime}$ in $H$ of length at most $d_{2}$. Without loss of generality we can assume $m \geq n$. We have

$$
\begin{aligned}
&(u, v) \rightarrow\left(u_{2}, v_{2}\right) \rightarrow\left(u_{3}, v_{3}\right) \rightarrow \cdots \rightarrow\left(u_{n}, v_{n}\right) \rightarrow \\
& \quad\left(u_{n+1}, v_{n}\right) \rightarrow \cdots \rightarrow\left(u_{m}, v_{n}\right)=\left(u^{\prime}, v^{\prime}\right)
\end{aligned}
$$

is a path of length at most $d_{1}$.

Since $\operatorname{diam}_{\min }\left(C_{3}\right)=2$ and $\operatorname{diam}_{\min }\left(C_{3} \otimes C_{3}\right)=2$, the bound is tight.

\section{References}

[1] V. Chvátal and C. Thomassen, Distances in orientations of graphs, J. Comb. Theory Ser. B 24 (1978), 61-75, doi:10.1016/0095-8956(78)90078-3.

[2] R. Hammack, W. Imrich and S. Klavžar, Handbook of Product Graphs, Discrete Mathematics and its Applications, CRC Press, Boca Raton, Florida, 2nd edition, 2011, doi:10.1201/b10959.

[3] J. S.-T. Juan, C.-M. Huang and I. Sun, The strong distance problem on the Cartesian product of graphs, Inform. Process. Lett. 107 (2008), 45-51, doi:10.1016/j.ipl.2008.01.001.

[4] K. M. Koh and E. G. Tay, On optimal orientations of Cartesian products of even cycles and paths, Networks 30 (1997), 1-7, doi:10.1002/(sici)1097-0037(199708)30:1<1::aid-net1〉3.0.co; 2-h.

[5] K. M. Koh and E. G. Tay, Optimal orientations of products of paths and cycles, Discrete Appl. Math. 78 (1997), 163-174, doi:10.1016/s0166-218x(97)00017-6.

[6] K. M. Koh and E. G. Tay, On optimal orientations of Cartesian products of graphs (II): complete graphs and even cycles, Discrete Math. 211 (2000), 75-102, doi:10.1016/s0012-365x(99) 00136-3.

[7] K. M. Koh and E. G. Tay, On optimal orientations of Cartesian products of trees, Graphs Combin. 17 (2001), 79-97, doi:10.1007/s003730170057.

[8] J.-C. Konig, D. W. Krumme and E. Lazard, Diameter-preserving orientations of the torus, Networks 32 (1998), 1-11, doi:10.1002/(sici)1097-0037(199808)32:1<1::aid-net1〉3.3.co;2-a.

[9] H. E. Robbins, Questions, discussions, and notes: A theorem on graphs, with an application to a problem of traffic control, Amer. Math. Monthly 46 (1939), 281-283, doi:10.2307/2303897.

[10] F. S. Roberts and Y. Xu, On the optimal strongly connected orientations of city street graphs I: Large grids, SIAM J. Discrete Math. 1 (1988), 199-222, doi:10.1137/0401022. 